\title{
Scaling SOI photonics to micron and sub-micron devices
}

\author{
Paolo Dainesi ${ }^{1,2}$, Kirsten Moselund $^{2}$, Marco Mazza $^{2}$, Luc Thévenaz $^{1}$ and Adrian Ionescu ${ }^{2}$ \\ 1 Nanophotonics and metrology laboratory \\ 2 Electronics Laboratory \\ Ecole Polytechnique Fédérale de Lausanne EPFL, 1015 Lausanne, Switzerland.
}

\begin{abstract}
Scaling photonics devices in silicon on insulator (SOI) substrates has the potential to address important issues in the fields of optical telecommunications and optical interconnects. Silicon, is highly transparent in the infra-red spectral region and etching ribs or rectangular channels can create the condition for single-mode low-loss waveguiding. The high index difference between silicon and the surrounding media, typically $\mathrm{SiO}_{2}$ or air, is extremely favorable for the development of ultra-compact photonic devices. Active functionality can be performed by free charge injection in the waveguide resulting in a phase shift of the propagating fundamental mode. Moreover this technology is fully CMOS compatible allowing a low-cost monolithic integration of control electronics. Limitations deriving from an aggressive scaling of SOI waveguides are a lowered efficiency in the in-out coupling of light and higher propagation losses due to increased roughness scattering.

We report on the perspectives and issues of scaling SOI photonics devices for both passive and active functionality. Results show that scaled waveguides can have very low bending radii down to the micrometer range. We also propose a new method and architecture for light phase modulation based on a Schottky barrier diode; a process flow will be analyzed and validated experimentally.
\end{abstract}

Keywords: silicon optoelectronics, photonic wires, scaled waveguides, optical switch, charge injection, optical interconnects, optical clock distribution, Schottky diode.

\section{INTRODUCTION}

Next generation photonics require active switching to achieve advanced functionalities and a proper processing of the optical signal. Ideally the switching time should be much below $1 \mu$ s for many present and future applications. Existing devices are either slow (MEMS, silica on silicon) or expensive ( $\mathrm{LiNbO}_{3}, \mathrm{III}-\mathrm{V}$ semiconductors), and their co-integration with control logics is still an open issue.

To simultaneously address these challenges, silicon-on-insulator (SOI) is a very promising substrate. Several research works have shown positive results in the last years ${ }^{1-4}$ concerning waveguides in SOI; the approach used for those purposes was the etching of a rib structure in the crystalline silicon film of a SOI wafer allowing for large waveguides with dimensions in the 5 to $10 \mu \mathrm{m}$ range (see fig. 1). These waveguides are single-mode provided that the following geometrical approximated condition is established: ${ }^{5}$

$$
\begin{aligned}
& 0.5<r<1 \\
& \frac{W}{H}<0.3+\frac{r}{\sqrt{1-r^{2}}}
\end{aligned}
$$

In order to achieve active operation both thermal effect and plasma dispersion effect (injection of free carriers) have been largely investigated in the past ${ }^{6-9}$ and both have shown their limitations: the first one is clearly appropriate only to slow applications and the second one, which is typically based on p-i-n diode injection is in principle limited by the recombination time (see figure 2). Among the best published results are modulators in the $10 \mathrm{MHz}$ range. 


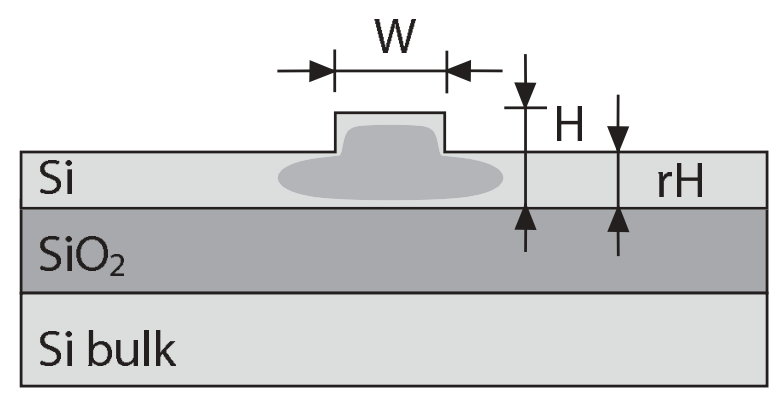

Figure 1. SOI rib waveguide. Single-mode propagation can be granted with specific restrictions on the geometrical parameters. Part of the fundamental optical mode propagates in the infinite slab below the waveguide rib.

More recently the first device based on a capacitive effect ${ }^{10}$ has been presented showing a record modulation frequency of $1 \mathrm{GHz}$; though this device clearly indicated a new possible very fast modulation principle, its suffers from rather low efficiency due to the poor overlap between the propagating optical field and the surface of the cumulated charge.

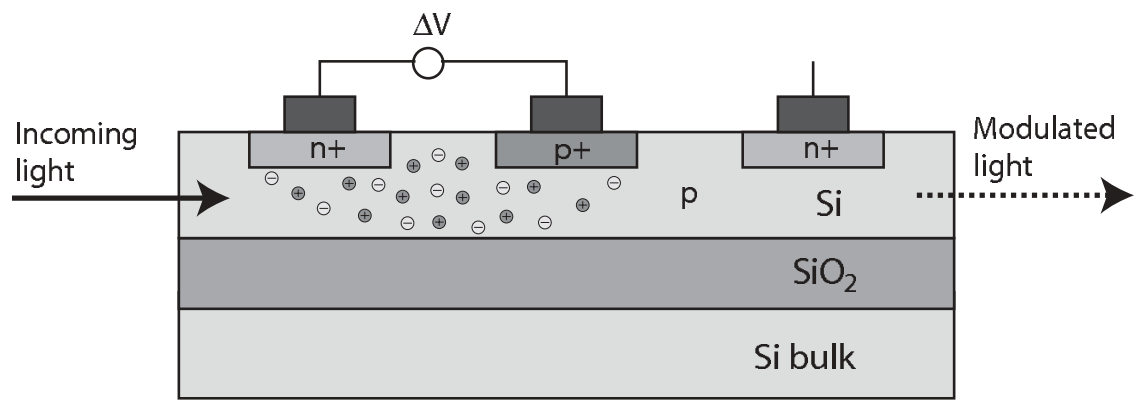

Figure 2. Schematic principle of a transversal p-i-n diode plasma dispersion effect light phase modulator in SOI.

Sub-micron waveguides, also photonics wires (PW), in silicon technology have also been investigated ${ }^{11-13}$ and results are very promising; single-mode propagation can be obtained in etched rectangular waveguides, allowing extremely high index difference between the propagating media and the surrounding cladding. Figure 3 shows typical SOI PWs in deep or shallow etching configuration respectively.

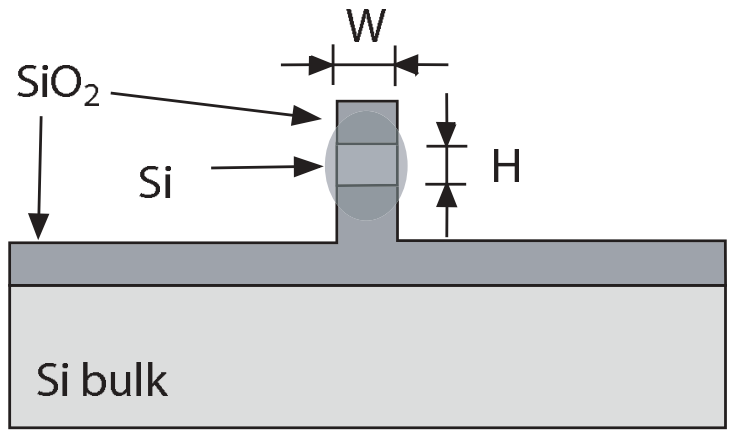

a)

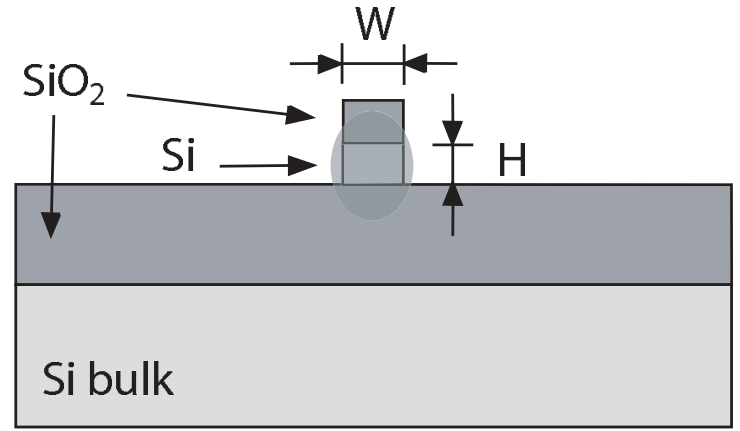

b)

Figure 3. SOI sub-micron waveguides (photonic wires). a) Deep etch. b) Shallow etch.

It has been shown ${ }^{14}$ that shallow etching can be useful for reducing the residual surface roughness. On such small waveguides, in fact, the surface roughness becomes the dominant propagation loss limitation and a specific surface smoothing process is required to reduce it to acceptable valuables. In recent publications this method has been used to show propagation losses as low as $2.4 \mathrm{~dB} / \mathrm{cm}$ in ref. 11 and $0.8 \mathrm{~dB} / \mathrm{cm}$ in ref. 15 . A record value of $0.5 \mathrm{~dB} / \mathrm{cm}$ has been shown with a numerical study ${ }^{16}$ in the smallest PW considered up to date, having $H=W=150 \mathrm{~nm}$. 
Very interesting engineering solutions have also been proposed for efficient light in/out coupling from a standard optical fiber to sub-micron silicon waveguides, the most promising are grating couplers ${ }^{17}$, silicon based tapers ${ }^{18,19}$ and polymer mode size converters ${ }^{20}$.

Scaling SOI waveguides to sub-micron dimensions has raised new very interesting challenges also in the field of nonlinear optics. The extremely high light intensities arising in such compact structures, can give rise to third order non linear phenomena, second order ones being absent due to the centro-symmetric silicon crystalline structure. The first encouraging results on this subject have been published very recently and pointed out Kerr effect ${ }^{21}$, two-photon absorption $^{22}$ and Raman spontaneous and stimulated scattering ${ }^{23-25}$ as the most interesting effects for future all-optical silicon active devices.

In this paper we show, by theoretical analysis and by numerical simulations, the effects of waveguide scaling to submicron dimension in SOI substrates on the device compactness and bend losses. We also propose a process flow for the fabrication of those waveguides which is comprehensive of a light phase modulator based on a Schottky barrier diode. Schottky diodes have recently found new applications within silicon optoelectronics, where they are used for their speed advantages in photo detectors ${ }^{26}$ or charge injection based light modulators ${ }^{27}$. A Schottky diode is advantageous with respect to scalability compared to a pn-diode, as the latter requires a diffused or implanted junction. Thus, Schottky diodes are more suited to sub-micron technologies. We will put in evidence critical fabrication points for the proposed Schottky phase modulator and discuss solutions together with preliminary measurement results.

\section{SOI WAVEGUIDES SCALING}

In rib waveguides (as shown in figure 1) very interesting performances have been demonstrated in the past, in particular in terms of high efficiency of light in/out coupling with standard optical fibers (especially for $H$ and $W>5 \mu \mathrm{m}$ ), due to the favorable mode matching, and also in terms of easiness of co-integration with standard CMOS electronics. These elements made of SOI rib-based waveguides a very promising platform for silicon optoelectronics.

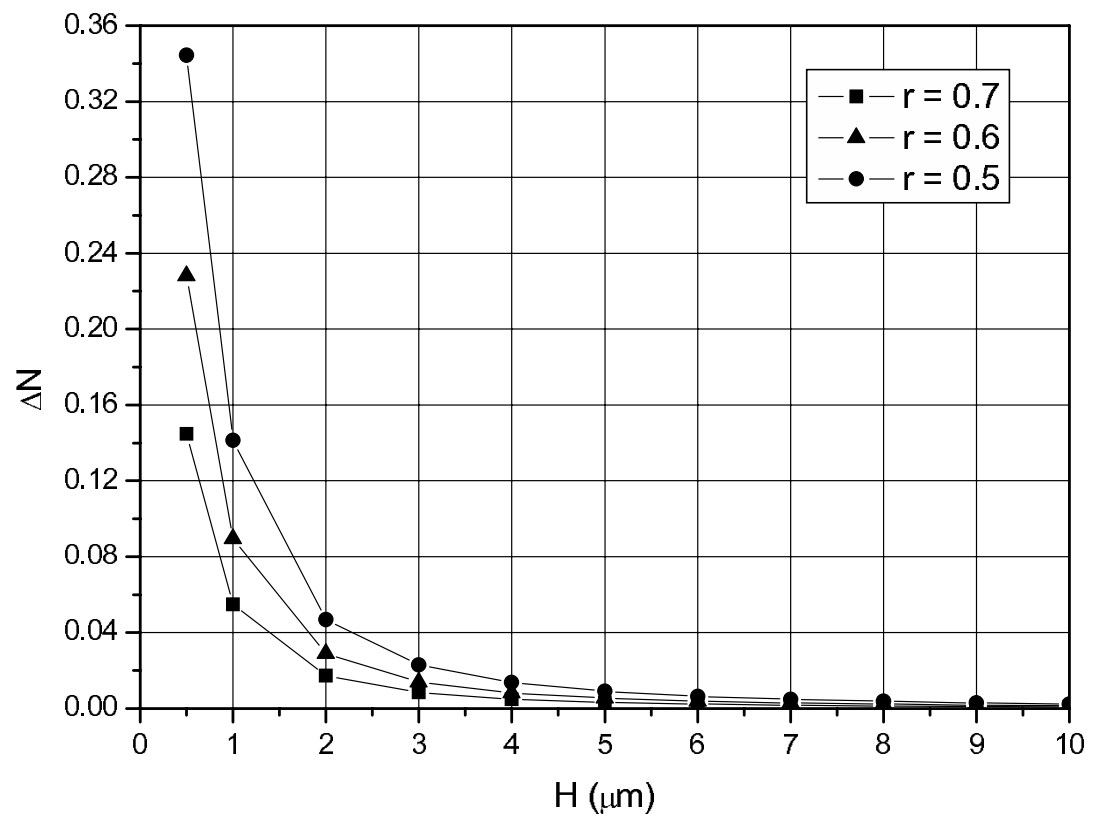

Figure 4. Index difference between the guiding layer and the cladding of the $2 \mathrm{D}$ equivalent model of rib waveguides for different height $H$ and etching ratio $r$. For each case the largest possible value of $W$ has been considered.

On the other side, single-mode operation in such large waveguides requires the waveguide design to satisfy equation (1), and, as a consequence, the rather low confinement of the propagating optical mode, due to the presence of the infinite 
silicon slab below the waveguide rib, is a serious limitation in the development of compact devices. With the use of the approximated effective index method we have calculated 2D models of rib waveguides for different values of height $H$ and etching ratio $r$, taking each time the highest possible width $W$. The resulting index difference $\Delta N$ between the waveguide medium and the cladding medium of the $2 \mathrm{D}$ model is presented in figure 4 . It appears that for $r=0.5$, corresponding to the situation in which the portion of the optical mode propagating in the infinite slab is the smallest, the index difference is higher and the propagating mode is more confined, consequently. It is also clearly visible in figure 4 that large ribs can have 2D equivalent index differences as low as $10^{-2}$ and shall than be considered as poorly suitable for complex functionalities. For $r=0.5$, then we have performed simulations of the fundamental optical mode for the equivalent 2D model waveguide for different values of the height $H$ and width $W$. Results are shown in figure 5, the index difference, effective index and confinement factor are also presented in the figure, showing that, in the singlemode propagation region the most scaled waveguides have the largest index difference (and hence the smallest exponential tail in the cladding). Moreover, it is possible to say that, for a given height, the largest possible waveguide is the one which has the higher confinement factor.

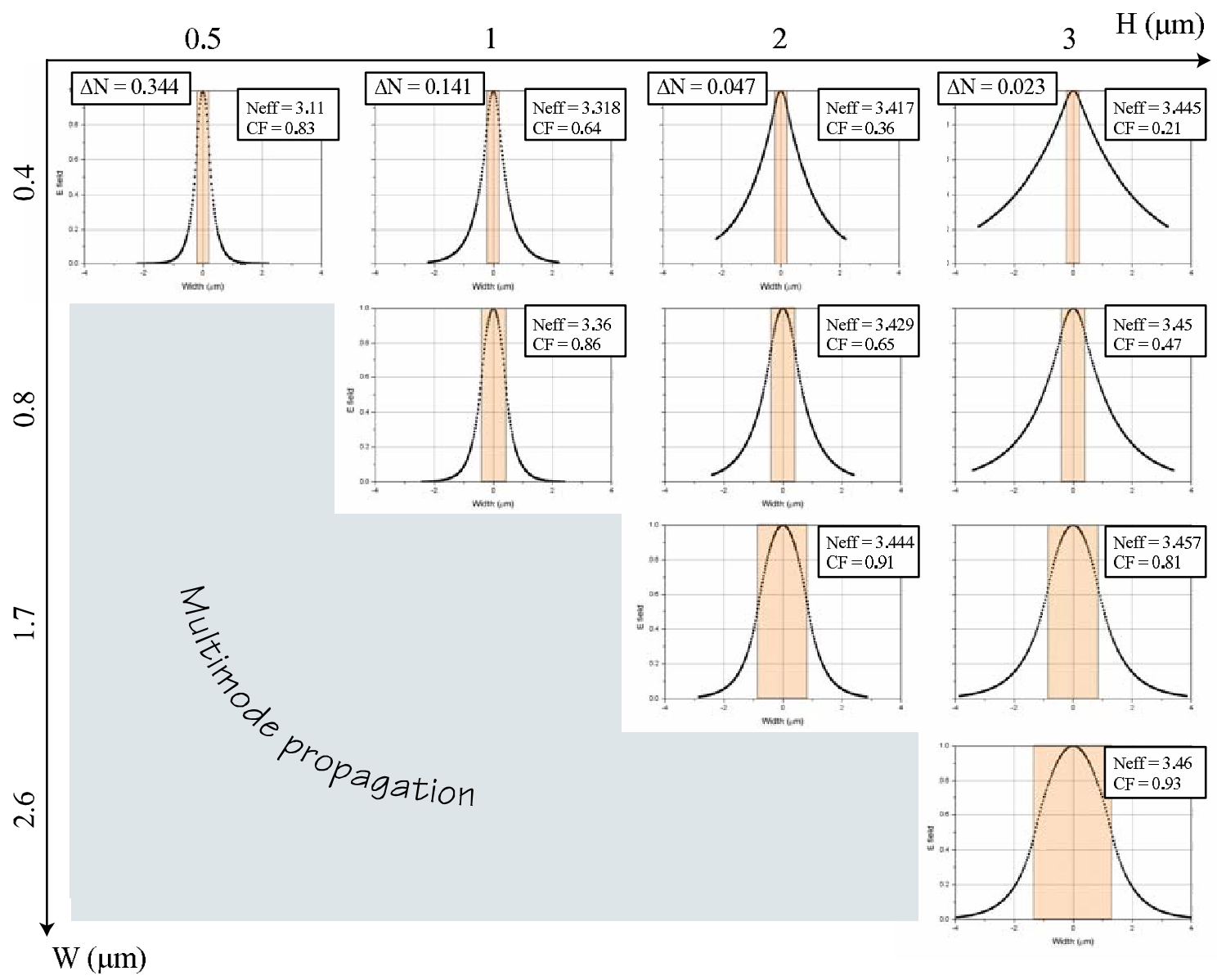

Figure 5. Simulated fundamental modes in 2D equivalent models of rib waveguides with different heights $H$. The index difference, confinement factor and modal index are calculated for each case.

Following Marcuse's theory ${ }^{29}$, the extent of the exponential tail of a propagating optical mode in the cladding medium is responsible for bending losses. It appears then clear from our previous analysis that only very small rib waveguides can have low bending radii. To validate this hypothesis we have calculated and plotted bend losses as a function of the bending radius, for different waveguide heights. For each curve the etching ratio is $r=0.5$ and the width is the widest possible with respect to the single-mode propagation condition. Results are shown in figure 6 . It is evident the large increment in bend losses from small rib waveguides to large rib waveguides; if bend losses are required to be inferior 
than $0.1 \mathrm{~dB} / \mathrm{cm}$, for example, bends can have radii as small as $150 \mu \mathrm{m}$ for $1 \mu \mathrm{m}$ height ribs, while radii larger than few centimeters are needed for ribs higher than $6 \mu \mathrm{m}$.

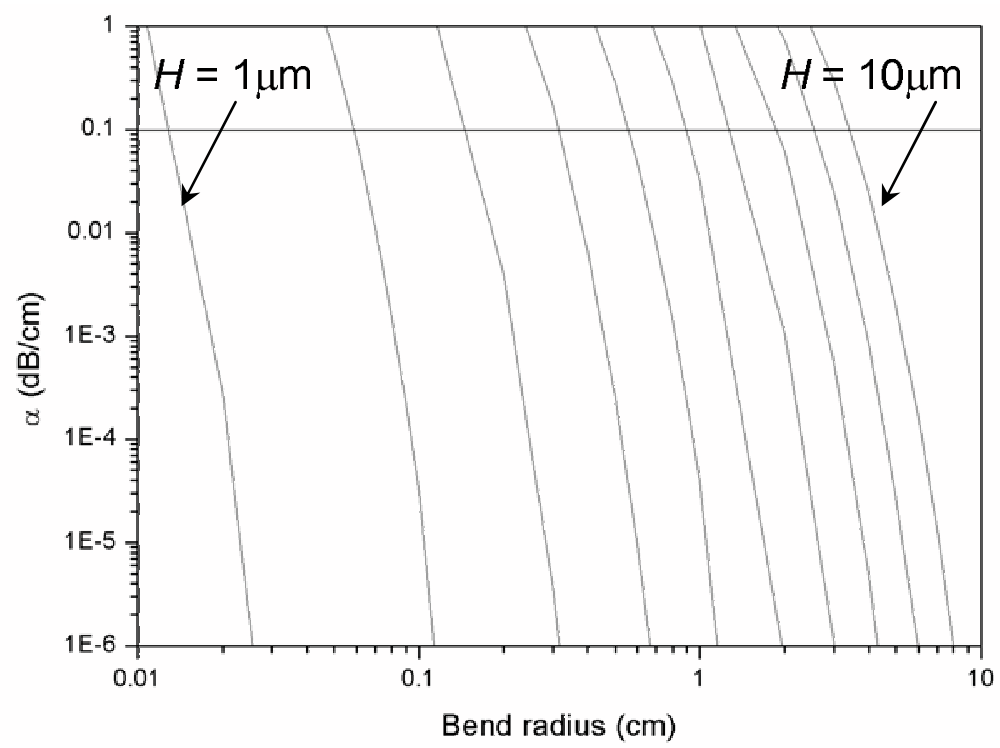

Figure 6. Bend losses for rib waveguides with height between $H=1 \mu \mathrm{m}$ and $H=10 \mu \mathrm{m}$ respectively plotted from left to right.

For each curve $r=0.5$ and $W$ is the largest possible respecting eq. (1).

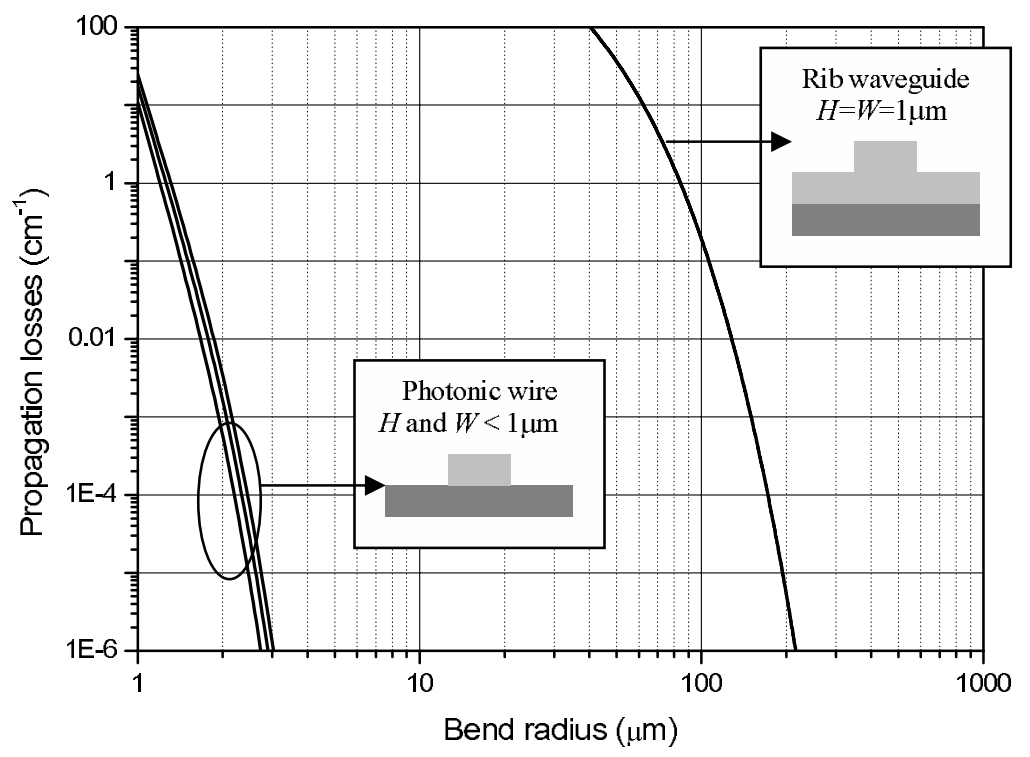

Figure 7. Bend losses comparison between small rib waveguides and photonic wires.

To be able to access smaller bending radii and hence extremely compact devices further scaling towards sub-micron photonic wires is required. In this case the single mode propagation condition can be obtained in rectangular channels, and, as a consequence, the resulting index difference will be much higher than any possible rib waveguide. It shall be pointed out also that since one of the key elements in SOI optoelectronics is its capability of co-integration with CMOS electronics, scaling towards thin sub-micron films is a fundamental element in providing valuable state of the art electronics on the same chip. CMOS electronics is evolving in the direction of fully depleted thin film and multiple gate configurations, and keeping pace with this trend, also from the optical point of view, will make possible co-integration of best performances in terms of both optical and electronic device progress. 




Figure 8. Zoomed portion of figure 7 evidencing the differences between the three different considered PWs.
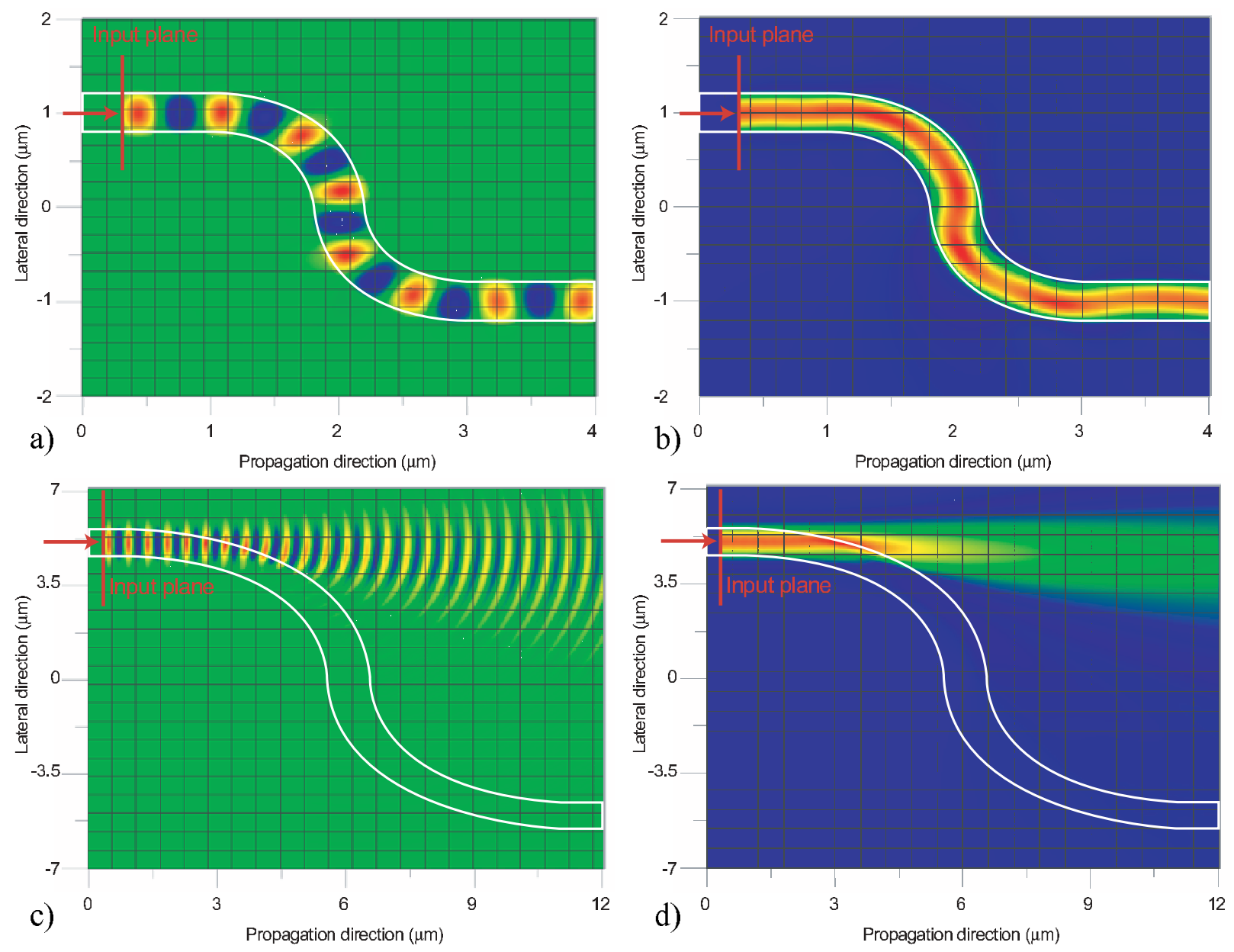

Figure 9. FDTD simulations of bends for PWs and rib waveguides. The white line sketches the simulated circuit. a) Time domain simulation for a PW with $H=200 \mathrm{~nm}$ and $W=500 \mathrm{~nm}$, bend radius is $R=1 \mu \mathrm{m}$. b) Filed amplitude for the device in a). c) Time domain simulation for a rib waveguide with $H=W=1 \mu \mathrm{m}, r=0.5, R=5 \mu \mathrm{m}$. d) Field amplitude for the device in c). 
To clearly show the difference between rib waveguides and photonic wires we have performed bend loss simulations with an FDTD algorithm in small PWs with different height and width (see figure 3) and compared them with the results for the $1 \mu \mathrm{m}$ high rib waveguide previously calculated. Results are shown in figure 7 and a zoomed portion of this graph is plotted in figure 8. It is clear from figure 7 and figure 8 that bending losses in the micrometer range are accessible for PWs and that while the differences between the three photonic wire geometrical configurations are quite modest, for given required bend losses, PWs can have two orders of magnitude lower bending radii than rib waveguides. This result is also confirmed by the FDTD simulations that we have performed on both PWs and rib waveguides, and presented in figure 9. The PW structure is a $200 \times 500 \mathrm{~mm}^{2}$ channel waveguide and the simulated circuit is composed of two symmetrical quarter arcs of circle with radius $R=1 \mu \mathrm{m}$, as sketched by the white line. Simulations in the time domain, figure $9 \mathrm{a}$ ) and of the field amplitude, figure $9 \mathrm{~b}$ ) put in evidence very modest losses in the range of $0.3 \mathrm{~dB}$. On the other hand, the simulated rib waveguide has $H=W=1 \mu \mathrm{m}, r=0.5$, and the simulated circuit is composed of two symmetrical quarters arcs of circles with radius $R=5 \mu \mathrm{m}$. It is evident both from the time domain simulation, figure $9 \mathrm{c}$ ) and from the simulated field intensity, figure $9 \mathrm{~d}$ ), that the propagating energy is entirely irradiated in the infinite slab below the rib. From these results it has been shown that a dramatic increase of performance in terms of compactness is expected from scaling further towards PW based SOI.

\section{SCHOTTKY DIODE PHASE MODULATOR}

We propose a fabrication process in order to develop Schottky-contact light phase modulators in small rib waveguides and photonic wires. The process flow for a small rib waveguide is presented in figure 10; with a single additional step this process can be adapted to sub-micron waveguides, provided the adequate lithography resolution. A hard mask is used to etch the waveguide structure, figure $10 \mathrm{a}$ ), then the ohmic contact of the diode is opened, and implanted, figure $10 \mathrm{~b}$ ). The surface treatment required for roughness smoothness is then applied, figure $10 \mathrm{c}$ ) and, finally, the Schottky barrier contact is opened and fabricated by metal deposition, figure $10 \mathrm{~d}$ ).

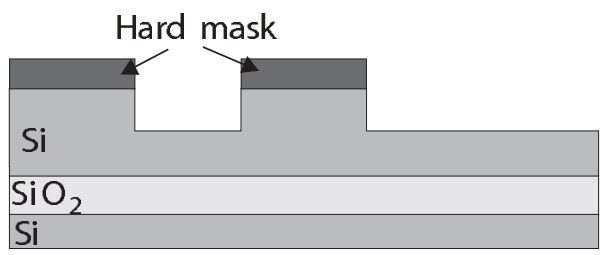

a)

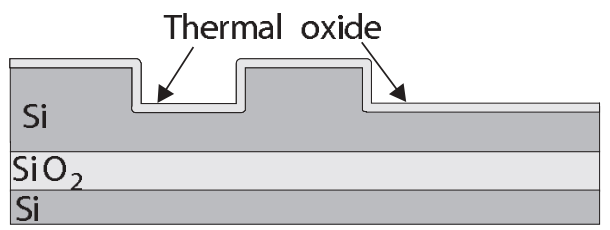

b)

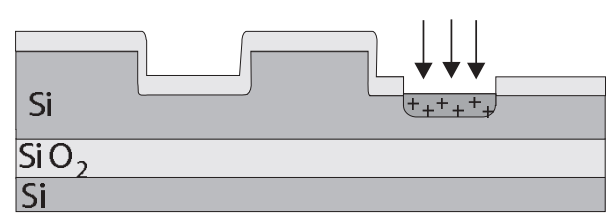

c)

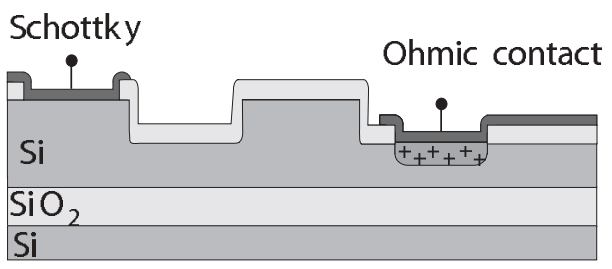

d)

Figure 10. Proposed process flow for the fabrication of Schottky diodes light phase modulators in micrometric rib waveguides and sub-micrometric photonic wires. a) Rib or channel etching. b) Surface roughness reduction.

c) Ohmic contact fabrication. d) Schottky contact fabrication.

The fabrication of good quality Schottky contacts is very sensitive to the different process parameters and hence, we have carried out an extensive study of Schottky diodes on silicon, using metals compatible with standard microelectronic processing, and having an approximately mid-gap barrier, which is suitable for optoelectronic purposes. Generally, good quality Schottky diodes and ohmic contacts were obtained, with currents in the mA range. It was found that the silicon surface preparation before the metal deposition is a critical point in the fabrication of Schottky diodes; standard RFetching cleaning before metal deposition, in fact, caused surface damage, which increased the barrier height as well as the contact resistance for Schottky diodes on p-type Si. This is in line with previous reports of the effect of surface damage by RIE etching ${ }^{28}$, where a positive charge at the metal semiconductor interface, was found to increase the barrier 
height on p-type diodes. In order to avoid a barrier shift and increased resistance we developed a process step especially conceived not to damage the Si surface before metallization (see figure 10 and 11); after dry etching of the waveguide, and consequent oxidization to reduce the surface roughness, immediately before metal deposition, we used an HF-dip to remove the native oxide. The benefit of this process step is visible in figure 12, which shows current-voltage measurements of Mo-Si Schottky contacts fabricated with three different process conditions. It is clear that the use of RF etch before Mo metallization is rather detrimental for the resulting resistivity of the diode contact, even though this negative effect can be partially recovered with the use of a post metal deposition anneal at $550^{\circ} \mathrm{C}$, on the contrary, the quality of the Mo-Si Schottky contact fabricated with the use of HF-dip immediately before Mo metallization is very good even without post-metallization anneal, which has shown to leave the diode characteristics unchanged in this case.



Figure 11. Scanning electron microscope picture of a fabricated Mo-Si Schottky contact.

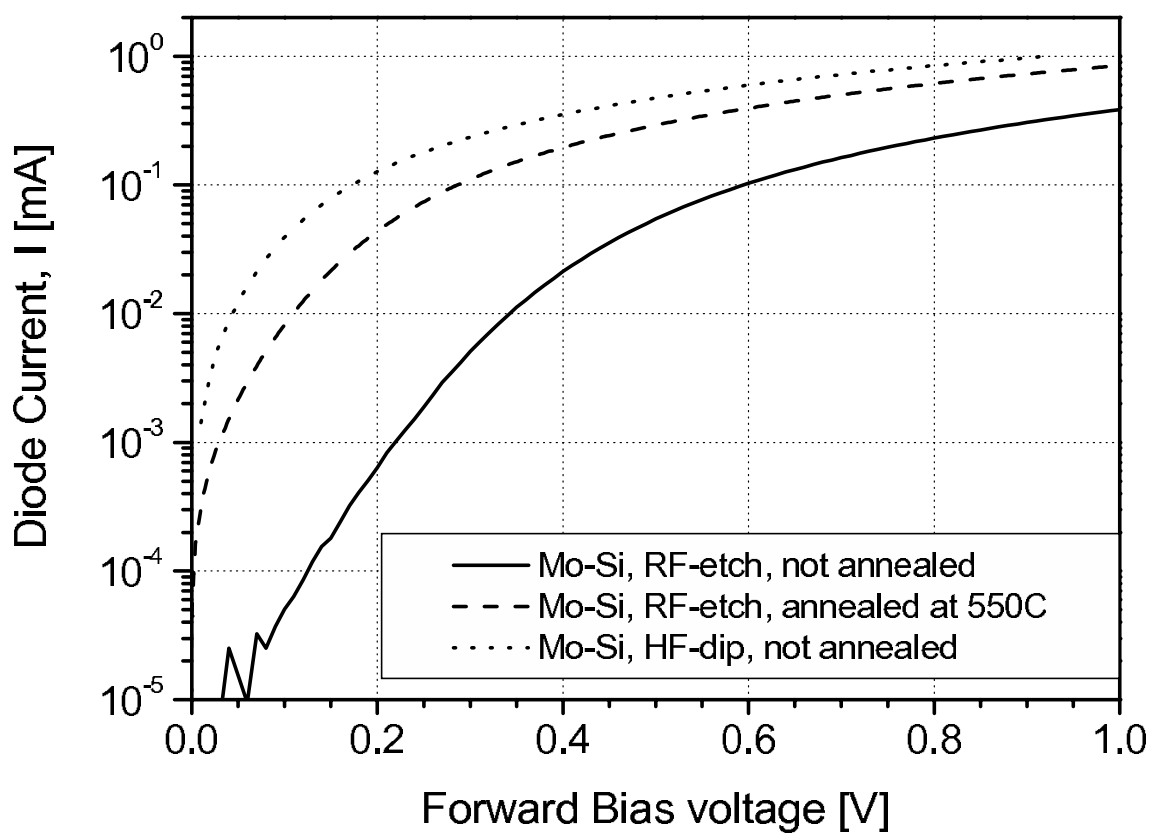

Figure 12. Current-Voltage characteristics of Mo-Si Schottky diodes fabricated with three different process steps, to put in evidence the issue of silicon surface cleaning before metal deposition. 


\section{CONCLUSION}

In conclusion we have shown by theoretical calculations and numerical analysis the rationale for scaling SOI optoelectronics towards very small ribs and photonic wires. Despite the requirements for deep-UV or e-beam lithography, an increase in the complexity of the process and the need to develop efficient in/out light couplers, the advantages in terms of compactness and hence functionality density makes this technology very attractive for the development of a new class of optoelectronic devices with co-integrated state of the art CMOS electronics, capable to address challenging issues in the field of optical telecommunications and on-chip clock/signal distribution.

Moreover, we have proposed a process flow in order to develop active devices in SOI based on a Schottky diode light phase modulator. Fabrication of devices based on this process are on going in a small rib architecture, but the process is easily modifiable for photonic wires. We have put in evidence a typical issue in the fabrication of Schottky diodes caused by the silicon surface damage before metallization and found and validated experimentally an appropriate solution.

\section{REFERENCES}

1. R. A. Soref and J. P. Lorenzo, "Single-Crystal Silicon: a New Material for 1.3 and $1.6 \mu \mathrm{m}$ Integrated Optical Components" Electronic Letters, Vol. 21, No. 21, 953 - 954, 1985.

2. J. Schmidtchen, A. Splett, B. Schüppert, K. Petermann "Low Loss Singlemode Optical Waveguides with Large Cross-Section in Silicon-on-Insulator", Electronic Letters, Vol. 27, No. 16, 1486 - 1488, 1991.

3. U. Fischer, T.Zinke, K. Petermann "Integrated Optical Waveguide Switches in SOI", IEEE Proceedings of the International SOI Conference, 141 - 142, 1995.

4. P. Dainesi, L. Thévenaz and Ph. Robert, "5 MHz 2x2 Optical Switch in Silicon on Insulator Technology Using Plasma Dispersion Effect", Proceeding of 27th European Conference on Optical Communications, ECOC 01, 2001.

5. K. Petermann "Properties of Optical Rib-Guides with Large Cross-Section" Archiv fur Electronik und Ubertragungstechnik, Vol. 30, 139 - 140, 1976.

6. G. Cocorullo, I. Rendina "Thermo-Optical Modulation at $1.5 \mu \mathrm{m}$ in Silicon Etalon", Electronics Letters, Vol. 28, No. $1,83-85,1992$.

7. G. V. Treyz "Silicon Mach-Zehnder Waveguide Interferometers Operating at $1.3 \mu \mathrm{m}$ ", Electronic Letters, Vol. 27 , No. $2,118-120,1991$.

8. C. K. Tang, G. T. Reed "Highly Efficient Optical Phase Modulator in SOI Waveguides", Electronic Letters, Vol. 31, No. 6, 451-452, 1995.

9. Y. L. Liu, E. K.Liu, S. L.Zhang, G.Z.Li, J.S. Luo, "Silicon $1 \times 2$ Digital Optical Switch Using Plasma Dispersion", Electronic Letters, Vol. 30, No. 2, 130 - 131, 1994.

10. A. Liu, R. Jones, L. Liao, D. Samara-Rubio, D. Rubin, O. Cohen, R. Nicolaescu and M. Paniccia, "A high-speed silicon optical modulator based on a metal-oxide-semiconductor capacitor", Nature, 427, 615 - 618, 2004.

11 P. Dumon, W. Bogaerts, W. Viaux, J. Wouters, S. Beckx, J. Van Campenhout, D. Taillaert, B. Luyssaert, P. Bientsman, D. Van Thourhout and R. Baets, "Low-Loss SOI Photonic Wires and Ring Resonators Fabricated with Deep UV Lithography", IEEE Photonics Technology Letters, Vol. 16, No. 5, 1328 - 1330, 2004.

12 C. A. Barrios, V.R. Almeida, R. Panepucci and M. Lipson, "Electrooptic Modulation of Silicon-on-Insulator Submicrometer-Size Waveguide Devices", IEEE Journal of Lightwave Technology, Vol. 21, No. 10, 2332 - 2339, 2003.

13 B. E. Little, J. S. Foresi, G. Steinmeyer, E. R. Thoen, S. T. Chu, H. A. Haus, E. P. Ippen, L. C. Kimerling and W. Greene, "Ultra-Compact $\mathrm{Si}_{-} \mathrm{SiO}_{2}$ Microring Resonator Optical Channel Dropping Filters", IEEE Photonics Technology Letters Vol. 10, No. 4, 549 - 551, 1998.

14 R. Baets, "Nanophotonics" Proceedings of the European Conference on Optical Communications ECOC 2003, Paper Tu 3.3, 2003.

15 K. K. Lee, D. R. Lim, L. C. Kimerling, J. Shin and F. Cerrina, "Fabrication of ultralow-loss $\mathrm{Si} / \mathrm{SiO}_{2}$ waveguides by roughness reduction", Optics Letters, Vo. 26, No. 23, 1888 - 1890, 2001.

16 F. Grillot, L. Vivien, S. Laval, D. Pascal and E. Cassan, "Size Influence on the Propagation Loss Induced by Sidewall Roughness in Ultrasmall SOI Waveguides", IEEE Photonics Technology Letters, Vol. 16, No. 7, 1661 1663, 2004.

17 G. Z. Masanovic, V. M. N. Passaro and G. T. Reed, "Dual Grating-Assisted Directional Coupling Between Fibers and Thin Semiconductor Waveguides", IEEE Photonics Technology Letters, Vol. 15, No. 10, 1395 - 1397, 2003. 
18 L. Vivien, S. Laval, E. Cassan, X. Le Roux and D. Pascal, "2-D Taper for Low-Loss Coupling Between Polarization-Insensitive Microwaveguides and Single-Mode Optical Fibers" IEEE, Journal of Lightwave Technology, Vo. 21, No. 10, 1 - 5, 2003.

19 V. R. Almeida, R. R. Panepucci and M. Lipson, "Nanotaper for compact mode conversion", Optics Letters, Vol. 28, No. 15, $1302-1304,2003$.

20 T. Shoji T. Tsuchizawa. T. Watanabe, K. Yamada and H. Morita, "Low loss mode size converter from $0.3 \mu \mathrm{m}$ square Si wire waveguides to singlemode fibers", Electronics Letters, Vol. 38, No. 25 1669-1670, 2002.

21 G. Priem, I. Notebaert, B. Maes, P. Bienstman, G. Morthier and R. Baets, "Design of All-Optical Nonlinear Functionalities Based on Resonators", IEEE Journal of Selected Topics in Quantum Electronics, Vol. 10, No. 5, $1070-1078,2004$.

22 V. R. Almeida, C. A. Barrios, R. R. Panepucci and M. Lipson, "All-optical control of light on a silicon chip", Nature, Vol. 431, 1081 - 1084, 2004.

23 H. Rong, R. Jones, A. Liu, O. Cohen, D. Hak, A. Fang and M. Paniccia, "A continuous-wave Raman silicon laser", Nature, Vol. 433, 725 - 728, 2005.

24 V. Raghunathan, D. Dimitropoulos, R. Claps and B. jalali, "Raman induced wavelength conversion in scaled Silicon waveguides", IEICE, Electronics Express, Vol. 1, No. 11, 298 - 304, 2004.

25 O. Boyraz and B. Jalali, "Demonstration of a silicon Raman Laser", Optics Express, Vol. 12, No. 21, 5269 - 5273, 2004.

26 Ch. Buchal, M. Löken, Th. Lipinsky, L. Kappius, and S. Mantl , "Ultrafast silicon based photodetectors", J. Vac. Sci. Technol. A, Vol. 18, No. 2, 630 - 634, 2000.

27 M. Mazza, L Thévenaz, P. Robert, M. Declercq and A. M. Ionescu, "A Novel Schottky Electro-optical Modulator for GHz High-speed Switching", Proceedings of the 12th International Conference on Solid State Sensors, Transducers '03, 1490 - 1493, 2003.

28 X. C. Mu and S. J. Fonash, "High-Barrier Schottky Diodes on p-type Silicon Due to Dry-Etching Damage", IEEE Electron Device Letters, Vol. EDL-6, No. 8, 410 - 412, 1985.

29 D. Marcuse "Light Transmission Optics", Second Edition, Van Nostrand Reinhold, 1982. 